\title{
QUADRATIC SUBFIELDS OF QUARTIC EXTENSIONS OF LOCAL FIELDS
}

\author{
JOE REPKA \\ Mathematics Department \\ University of Toronto \\ Toronto, Ontario \\ CANADA M5S IAI
}

(Received March 18, 1987)

\begin{abstract}
We show that any quartic extension of a local field of odd residue characteristic must contain an intermediate field. A consequence of this is that local fields of odd residue characteristic do not have extensions with Galois group $\mathrm{A}_{4}$ or $\mathrm{S}_{4}$. Counterexamples are given for even residue characteristic.
\end{abstract}

KEY WORDS AND PHRASES. Local field, quartic extension, endoscopic group. 1980 AMS SUBJECT CLASSIFICATION CODES. $12 B 2512 \mathrm{~B} 27$.

Research supported by the Natural Sciences and Engineering Research Council of Canada.

1. INTRODUCTION.

In Section 2, a simple application of local class field theory proves the existence of intermediate fields for quartic extensions of local fields with odd residue characteristic. This immediately implies the non-existence of Galois extensions of type $\mathrm{A}_{4}$ or $\mathrm{S}_{4}$ over such fields.

In Section 3, examples are given of $\mathrm{A}_{4}$ and $\mathrm{S}_{4}$ extensions of fields with even residue characteristic, and of a quartic extension with no intermediate field.

In Section 4, the results of Section 2 are used to show that the splitting field of an irreducible quartic polynomial over a local field must have degree 4 or 8 , provided the residue characteristic is odd. The implications of the results of Section 2 and Section 3 for the theory of endoscopic groups are also discussed.

I wish to thank Noriko Yui for helpful conversations about this work.

2. EXISTENCE OF INTERMEDIATE EXTENSIONS.

Let $F$ be a non-archimedean local field. Let $0=o_{F}$ and $p=p_{F}$, respectively, be the ring of integers of $F$ and its prime ideal.

THEOREM 2.1. Suppose the residue characteristic of $F$ is odd, and $E / F$ is a quartic extension (i.e. $[E: F]=4$ ). Then there must be an intermediate field $K$, i.e. $\mathrm{E} \supset \mathrm{K} \supset \mathrm{F},[\mathrm{E}: \mathrm{K}]=[\mathrm{K}: \mathrm{F}]=2$. 
PROOF: If $E / F$ is unramified, the result is obvious. If the ramification index of $E / F$ is $e=2$, then we must have $f=2$ and, by Corollary 4 to Theorem 7 of chapter I, Section 4 of Weil [1], there is an unramified quadratic intermediate field.

Now suppose $e=4$, so $f=1$. Any unit in $E$ is of the form $u+p$, with $u \in o_{F}^{\times}$and $p \in p_{E}$. The norm of such an element is $u^{4}+p^{\prime}$, with $p^{\prime} \in p_{E} \cap F=p_{F}$. So by Hensel's Lemma the only units contained in the image of $\mathrm{N}_{E / F}$ are fourth powers. In particular, $\mathrm{N}_{E / F}$ is not surjective, so Corollary 1 to Theorem 4 of chapter XII, Section 3 of Weil [1] proves the theorem.

Translating this into the corresponding result on Galois groups, we obtain the following equivalent formulation ...

THEOREM 2.2. If $F$ has odd residue characteristic, there cannot be a Galois extension $\mathrm{E} / \mathrm{F}$ whose Galois group is isomorphic to $\mathrm{A}_{4}$ or $\mathrm{S}_{4}$.

PROOF: $\mathrm{A}_{4}$ contains subgroups of index 4 (the cyclic group generated by any 3-cycle), none of which is properly contained in any proper subgroup (such a proper subgroup, if it existed, would be of order 6 and index 2, hence normal, hence would contain all 3-cycles, of which there are 8).

An $\mathrm{S}_{4}$-extension of $\mathrm{F}$ would be an $\mathrm{A}_{4}$-extension of a quadratic extension of $F$.

3. COUNTEREXAMPLE FOR RESIDUE CHARACTERISTIC 2.

Let $F=Q_{2}$ and consider the Eisenstein polynomial $\Phi(X)=X^{4}-2 X-2 \in F[X]$. Let $\mathrm{E}$ be the splitting field of $\Phi(X)$; we shall show that $\mathrm{Gal}(\mathrm{E} / \mathrm{F})=\mathrm{S}_{4}$ and $\mathrm{Gal}(\mathrm{E} / \mathrm{K})=\mathrm{A}_{4}$, where $K=Q_{2}(\sqrt{3})$. In the process we shall find a quartic extension $\mathrm{L} / \mathrm{F}$ with no intermediate field.

Let $\alpha$ be a root of $\Phi(X)$, and let $L=F(\alpha)$.

LEMMA 3.1. The norm $\mathrm{N}_{\mathrm{L} / \mathrm{F}}$ is surjective.

PROOF: Notice that $N(\alpha+1)=\Phi(-1)=1, N(\alpha-1)=\Phi(1)=-3$. Also the characteristic polynomial of $\alpha^{3}$ is $\Phi_{3}(\mathrm{X})=\mathrm{x}^{4}-6 \mathrm{X}^{3}+12 \mathrm{X}^{2}-8 \mathrm{X}-8$, so $N\left(\alpha^{3}+1\right)=\Phi_{3}(-1)=19$. If $N=N_{L / F}$ were not surjective, its image would be contained in the image of the norm map from some ramified quadratic extension of $F$. Such an image contains exactly two of the four cosets of $\circ^{x}$ modulo $\left(0^{x}\right)^{2}$. We have just shown $N_{L / F}$ contains the three cosets containing $1,-3$, and 19 .

In particular (by Corollary 1 to Theorem 4 of chapter XII, Section 3 of Weil [1]), L/F is a quartic extension with no intermediate field.

Factoring the polynomial $\Phi(X)$ over L, we see that $\Phi(X)=(X-\alpha) \Psi(X)$, where $\Psi(X)=X^{3}+\alpha X^{2}+\alpha^{2} X+\left(\alpha^{3}-2\right)$.

PROPOSITION 3.2. $\Psi(\mathrm{X})$ is irreducible over $\mathrm{L}$.

PROOF: If all roots of $\Psi(X)$ were in $L$, then $L=E$ would be Galois, in contradiction of Lemma 3.1. The only other way for $\Psi(X)$ to be reducible would be for exactly one root, $\alpha^{\prime}$ say, to be in $L$. In this case, 
$F\left(\alpha^{\prime}\right)$ would be a quartic extension of $F$ contained in $L$, hence $F\left(\alpha^{\prime}\right)$ $=F(\alpha)=L$.

Let $\sigma \in \operatorname{Gal}(E / F)$ be such that $\sigma(\alpha)=\alpha^{\prime}$. Then $\sigma(F(\alpha))=F\left(\alpha^{\prime}\right)$, and $\alpha^{\prime} \in F(\alpha)$ implies that $\sigma\left(\alpha^{\prime}\right) \in F\left(\alpha^{\prime}\right)=F(\alpha)=L$. Since $\sigma\left(\alpha^{\prime}\right) \neq \alpha^{\prime}, \sigma\left(\alpha^{\prime}\right)$ must equal the only other conjugate of $\alpha^{\prime}$ in $L$, i.e. $\sigma\left(\alpha^{\prime}\right)=\alpha$. Hence the fixed field $L^{\sigma}$ contains $\alpha+\alpha^{\prime}$ and $\alpha \alpha^{\prime}$, so $(X-\alpha)\left(X-\alpha^{\prime}\right)$ $=x^{2}-\left(\alpha+\alpha^{\prime}\right) X+\alpha \alpha^{\prime} \in L^{\sigma}[X]$, which shows that $\alpha$ is quadratic over $L^{\sigma}$. So $\left[L: L^{\sigma}\right]=\left[L^{\sigma}: F\right]=2$. This also contradicts Lemma 3.1 .

So $E$ is the splitting field of $\Psi(X)$ over $L$, and $G a l(E / L)$ is either $\mathrm{A}_{3}$ or $\mathrm{S}_{3}$.

$$
\text { Now } \Psi(X)=X^{3}+\alpha X^{2}+\alpha^{2} X+\alpha^{3}-2=X^{\prime}+(2 / 3) \alpha^{2} X^{\prime}+(20 / 27) \alpha^{3}-2,
$$

where $X^{\prime}=X+2 / 3$. Hence the discriminant of $\Psi(X)$ is $27\left((20 / 27) \alpha^{3}-2\right)^{2}-4\left((2 / 3) \alpha^{2}\right)^{3}=4.27+(368 / 27) \alpha^{6}-80 \alpha^{3} \equiv 4.9 .3 \bmod *\left(1+4 p_{L}\right)$.

Since $4.9\left(1+4 p_{L}\right) \subset\left(L^{x}\right)^{2}$, the discriminant of $\Psi(X)$ is a square in $L$ if and only if 3 is.

LEMMA 3.3. The element 3 is not a square in $L$.

PROOF: If 3 were a square, truncation of its square root would give an element of the form $x=1+a \alpha+b \alpha^{2}+c \alpha^{3}$, with $a, b$, and $c$ each equal to 0 or $I$ and so that $3-x^{2} \in 4 p_{L}$. A trivial computation shows that this is impossible.

Accordingly $\mathrm{Gal}(\mathrm{E} / \mathrm{L})=\mathrm{S}_{3}, \mathrm{Gal}(\mathrm{E} / \mathrm{F})=\mathrm{S}_{4}$, and $\mathrm{Gal}(\mathrm{E} / \mathrm{K})=\mathrm{A}_{4}$, where $K=F(\sqrt{3})$.

4. APPLICATIONS.

1. The splitting field of a quartic polynomial over a local field is severely constrained by the results of Section 2 .

THEOREM 4.1. Let $F$ be a local field with odd residue characteristic. Let $f(X) \in F[X]$ be an irreducible polynomial with $\operatorname{deg} f(X)=4$. Let $E$ be the splitting field of $f(X)$ over $F$. Then $[E: F]=4$ or 8 .

PROOF: $\mathrm{Gal}(\mathrm{E} / \mathrm{F})$ is a subgroup of $\mathrm{S}_{4}$. But by Theorem 2.2 it cannot be $\mathrm{S}_{4}$ or $\mathrm{A}_{4}$. Since $4 \mid[\mathrm{E}: \mathrm{F}]$, the only possibilities are 4 or 8 .

The polynomial $\Phi(\mathrm{X})$ of Section 3 gives a counterexample to this result when the residue characteristic is 2 . Theorem 4.1 is clearly equivalent to Theorem 2.2 (and hence to Theorem 2.1).

2. If $F$ is a local field, let $G=S L(4, F)$, and let $T$ be an elliptic torus in $G$. To $T$ is associated a quartic extension $E / F$ so that the centralizer of $T$ in $G L(4, F)$ is isomorphic to $E^{x}$, and $T$ itself is isomorphic to $E_{I}^{X}=\left\{x \in E^{\times} ; N_{E / F}(x)=I\right\}$.

The theory of endoscopic groups (cf. Langlands [2], Shelstad [3]) associates to $G$ and $T$ some other groups, among which the most interesting are constructed as follows: let $\mathrm{E} \underset{\neq}{\supset} \underset{z}{\supset F}$ and let $G^{\prime}=\left\{g \in G L(2, K): N_{K / F}(\operatorname{detg})=I\right\}$. In $G^{\prime}$ it is possible to find an 
elliptic torus $T^{\prime}$ associated to the quadratic extension $E / K$, and there is an isomorphism between $T$ and $T^{\prime}$. The hope is to simplify calculations with orbital integrals over the G-conjugacy class of $t \in T$ by comparing them with orbital integrals over the G'-conjugacy class of the corresponding $t^{\prime} \in T^{\prime}$.

The example of Section 3 shows that this approach will not apply for certain tori when the residue characteristic is 2 ; happily, for these tori the ordinary orbital integrals are invariant under stable conjugacy, so the problem does not arise. The results of Sections 2 encourage optimism in the case of odd residue characteristic.

\section{REFERENCES}

1. WEIL, A. Basic Number Theory, 3rd Edition, Springer-Verlag (New York, Heidelberg, Berlin) 1974.

2. LANGLANDS, R.P. Les Débuts d'une Formule des Traces Stable, Publ. Math. de I'Université Paris VII (1983) 188p.

3. SHELSTAD, D. Orbital integrals and a family of groups attached to a real reductive group, Ann. Scient. Ec. Norm. Sup. 12 (1979), 1-31. 


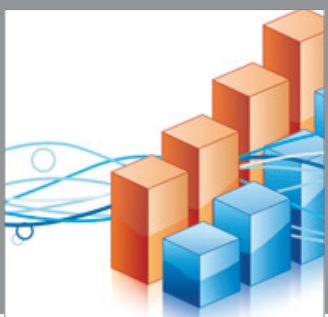

Advances in

Operations Research

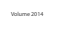

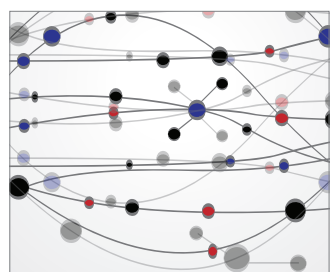

\section{The Scientific} World Journal
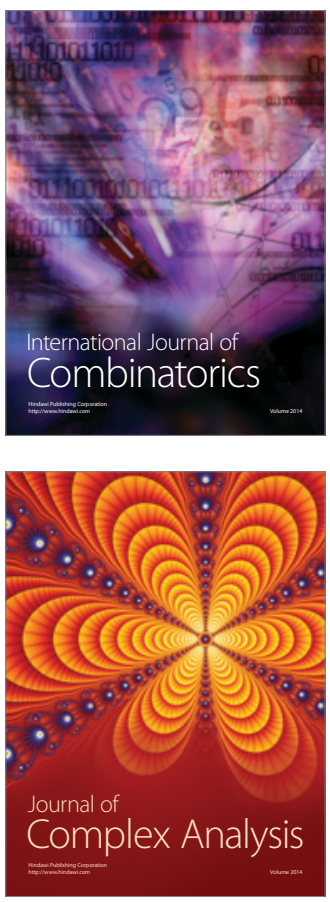

International Journal of

Mathematics and

Mathematical

Sciences
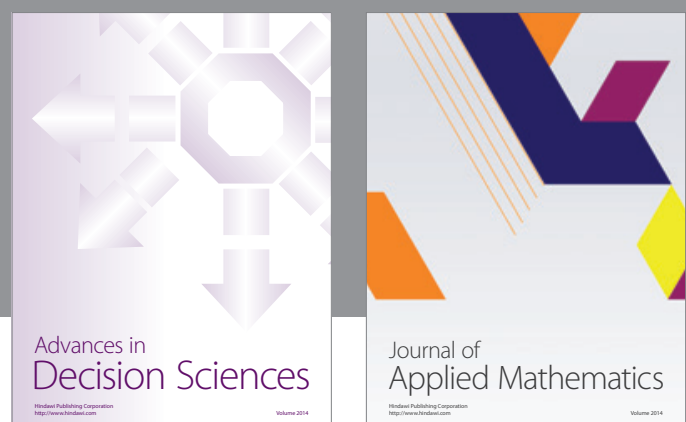

Journal of

Applied Mathematics
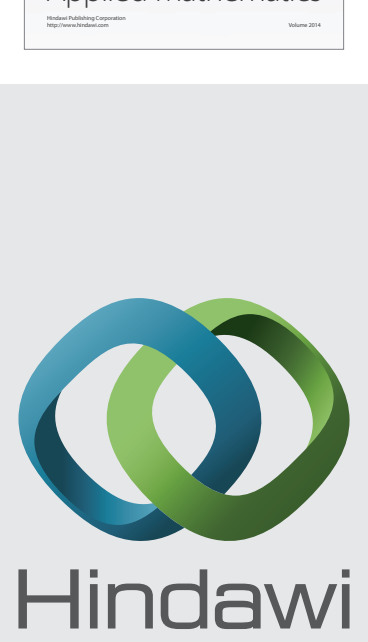

Submit your manuscripts at http://www.hindawi.com
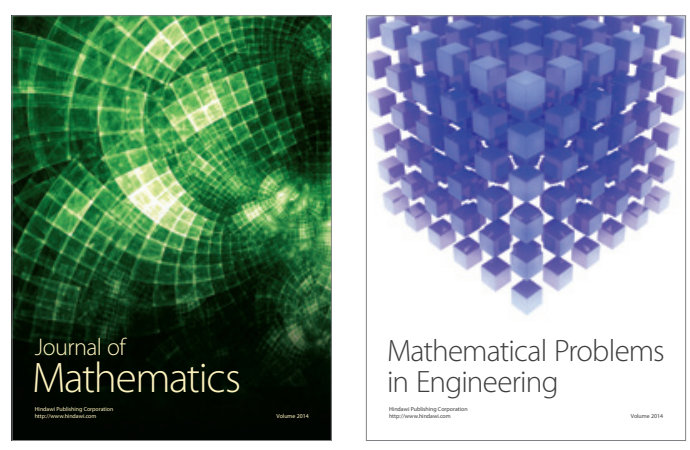

Mathematical Problems in Engineering
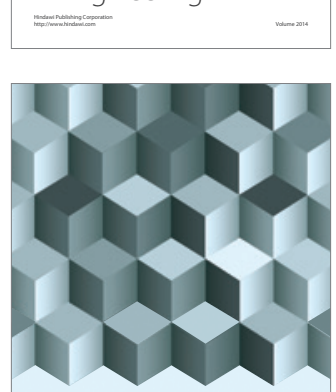

Journal of

Function Spaces
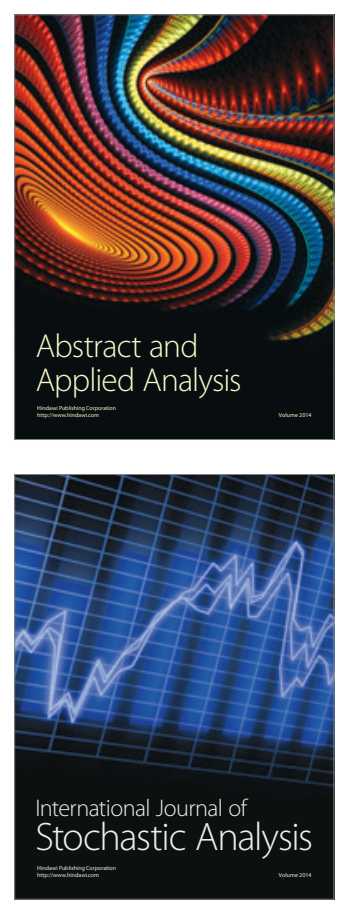

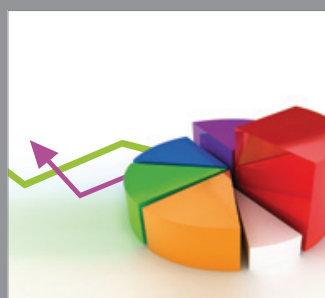

ournal of

Probability and Statistics

Promensencen
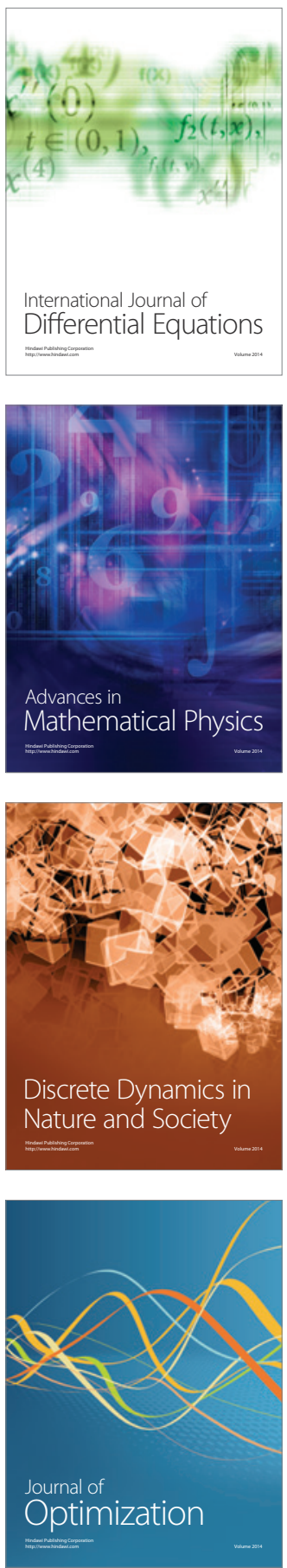